East African Medical Journal Vol. 85 No. 7 July 2008

LAPAROSCOPIC MYOMECTOMY OF A LARGE PEDUNCULATED FIBROID: CASE REPORT

R.B. Parkar, MBBS, MMed (Obs \& Gynae), FAGE, Dip. Lap. Surg., Consultant Obstetrician and Gynaecologist, P.O. Box 520-00606, Nairobi, Kenya, A. Chudasama and M. Chudasama, Consultant Obstetrician and Gynaecologist, P.O. Box 40088-80100, Mombasa, Kenya

Request for reprints to: Dr. R.B. Parkar, P.O. Box 520-00606, Nairobi, Kenya

\title{
LAPAROSCOPIC MYOMECTOMY OF A LARGE PEDUNCULATED FIBROID: CASE REPORT
}

\author{
R.B. PARKAR, A. CHUDASAMA and M. CHUDASAMA
}

\begin{abstract}
SUMMARY
A 37 year old para $0+0$ presented with abdominal pain and progressive abdominal swelling. She had no menstrual complains. Abdominal examination revealed a large 30 week non-tender, firm and mobile mass. A pelvic / abdominal ultrasound confirmed a $15.7 \times 9.9 \mathrm{~cm}$ pedunculated fibroid arising from the uterine fundus. A laparoscopic myomectomy was successfully undertaken, and the morcellated fragments weighed 1490 grams.
\end{abstract}

\section{INTRODUCTION}

Uterine fibroids are the most common benign neoplasms of the female genital tract, estimated to occur in $20-50 \%$ of women, with an increased frequency in the late reproductive age (1). Surgery remains the principal mode of treatment for these leiomyomata. The first reported laparoscopic myomectomy was by Semm and Mettler (2). Myomectomies were till then done primarily by laparotomy. They advocated careful client selection while undertaking laparoscopic myomectomy (2). Subsequently numerous reports of this technique have been published, establishing the effectiveness and safety of this technique in properly selected cases (3). These criteria include presence or absence of previous scars, number, location and size of fibroids. As myomectomy is done to preserve the uterus for future pregnancy, maintaining the integrity of the uterine wall during repair is of utmost importance. Difficulty in suturing after the myomectomy poses the major challenge, and three case reports of spontaneous uterine rupture in pregnancy after laparoscopic myomectomy have raised doubts regarding the safety of this technique on those still desiring fertility (5-7). The potential of thermal necrosis and wound dehiscence is also of concern (7).

\section{CASE REPORT}

A 37 year-old para $0+0$, presented with a history of lower abdominal pain and a progressive enlargement of the abdomen. Her cycles were normal. There was no history of any intermenstrual bleeding, dysuria or $\mathrm{p} v$ discharge.

There was no past medical history of diabetes, bronchial asthma or hypertension. She had undergone an appendicectomy in 1982. She was single, and worked as a corporate secretary. Her last normal menstrual period was on the 24th May 2007. The periods were moderate, with minimal clots, every 28 days for five to six days.

On general examination she was in fair general condition, well built and well nourished with adequate hydration. There was no pallor, cyanosis, icterus or any significant lymphadenopathy. She had no pedal or sacral oedema. The vital signs were stable, she was afebrile, and the blood pressure was elevated at admission. The breasts were not active. The cardiovascular, respiratory and the central nervous systems were clinically within normal. Abdominal examination revealed a previous appendicectomy scar. There was no tenderness. The liver and the spleen were not palpable. There was a large 30 week size non-tender, firm, mobile 
mass appreciated. There was no evidence of any intra-abdominal free fluid. The bowel sounds were normal.

On internal examination, the external genitalia were normal, vaginal mucosa healthy. The uterus was bulky in size, regular and anteverted. There was no adnexial tenderness or masses appreciated. However one large pedunculated mobile mass was evident. The pouch of douglas appeared empty. The cervix was firm, regular and appeared normal. Cervical excitation was negative. There was a minimal discharge on the examining finger.

A pelvic ultrasound done reported an anteverted uterus with a pedunculated fibroid measuring $15.7 \mathrm{x}$ $9.9 \mathrm{~cm}$ and two small intramural fibroids measuring $2 \mathrm{~cm}$ and $4.1 \times 2.9 \mathrm{~cm}$. Both the ovaries were not seen. The haemoglobin was $11.6 \mathrm{gms} / \mathrm{dl}$ and the urea and electrolytes were normal, blood sugar was 5.6 mmols/L. Her blood group was B positive.

A diagnosis of a large penduculated uterine fibroid was entertained and she opted for a hysteroscopy and laparoscopy with a possible laparotomy, after discussing all the available options.

In Theatre under fluid distention, at Hysteroscopy the uterine cavity was well visualised and appeared normal. Both the tubal ostia were seen and appeared normal. There were no submucous fibroids or endometrial polyps evident. The panoramic view was normal. An endometrial biopsy was obtained and sent for histology.

A routine pneumoperitonium was created. At laparoscopy a good view was obtained. The liver and gall bladder appeared normal. Both the ovaries and fallopian tubes were normal. The ovarian fossa, pouch of douglas and uterosacral ligaments were devoid of any endometriotic lesions. The uterus was bulky and with one large pedunculated fibroid (18 to $20 \mathrm{cms}$ in size) and two small $2-3 \mathrm{cms}$ fundal subserous fibroids.

A laparoscopic myomectomy was undertaken using the harmonic scalpel and bipolar coagulation. The endometrial cavity was not breeched. The defect was closed in a single layer using intracorporeal sutures. The fibroid was delivered by morcellation. A good lavage ensured adequate haemostasis. The urine was clear and postoperative observations were stable. The estimated blood loss was $200 \mathrm{mls}$. Bilateral ureteric peristalsis was evident. The specimens were sent for histological evaluation, which confirmed a lieomyoma. The weight of the dominant fibroid was 1490 grams.
She recovered well and was discharged in satisfactory condition. She has been subsequently reviewed every three months and is doing well.

\section{DISCUSSION}

Laparoscopic myomectomy is a safe and feasible procedure in appropriately selected patients. It is already shown in a series of reports that pregnancy rates between 36 and $65 \%$ can be expected following myomectomy done via laparotomy, in infertile women (8).

Such reports are now also seen following laparoscopic myomectomy (9). Myomectomy remains a challenging surgical procedure since it incorporates the reconstruction of an organ that usually undergoes significant structural modifications, as seen in pregnancy.

Laparotomic myomectomy has shown to be adequate with repair of such uteri, and normal reproductive performance thereafter (10). Only sporadic cases of pregnancy related uterine dehiscence have been reported following myomectomy by laparotomy (11).

Some of the main concerns regarding laparoscopic myomectomy have been adequate reconstruction and healing of the uterine wall defect following myomectomy, and the possible resulting obstetric complications, including ability of the uterus to withstand pregnancy and labour, and high incidence of elective Caesarean section (12). Many of the previous cases of uterine rupture in pregnant women following laparoscopic myomectomy occurred during second and third trimesters (28-36 weeks) $(12,13)$. Seracchioli et al advocated adequate tissue approximation, prevention of haematoma formation and limited use of electro coagulation as pointers towards a successful laparoscopic procedure (13). The patient presented was a 37 year old single female with no current desire of fertility.

At laparotomy, closure of the excision site is usually multilayered. With operative laparoscopy, this suturing can be tedious and cumbersome and thus the restoration of normal wall integrity can be challenging, especially with inexperienced hands. In the case under discussion a large pedunculated fibroid was encountered and intracorporeal suturing was successfully undertaken. The endometrial cavity was not breached. 
Marret et al (12) in their retrospective multicentre study noted that laparoscopic myomectomy be offered as a reproducible and safe alternative to abdominal myomectomy. The key factors for a successful outcome also include small myomas fewer in number, size, myoma location and the surgical expertise of the team. In our patient a single large pedunculated fibroid, 18 to $20 \mathrm{~cm}$ was encountered and managed successfully.

Seracchioli et al (13) in their evaluation on laparoscopic myomectomy reported that the feasibility of laparoscopic myomectomy has definitely been demonstrated. Although no definitive criteria have been established, deep intramural fibroids reaching the uterine cavity may represent a contraindication to laparoscopic myomectomy, due to intraoperative bleeding and inadequate closure of the myometrium.

In conclusion, laparoscopic myomectomy will gradually find its role in the management of patients with symptomatic uterine fibroids, as the surgical expertise improve.

\section{ACKNOWLEDGEMENTS}

To our colleagues and the administration and staff of the Mombasa Hospital, and to the patient for allowing us to publish this rather rare case.

\section{REFERENCES}

1. Verkauf, B.S. Myomectomy for fertility enhancement and preservation. Fertil. Steril. 1992; 58: 1-5.

2. Semm, K. and Mettler, L. New methods of pelviscopy for myomectomy, ovariectomy, tubectomy and adnexectomy. Endoscopy. 1979; 11: 85-93.

3. Dubuission, L.B., Lecuru, F., Foulot, H., et al. Myomectomy by laparoscopy: A preliminary report of 43 cases. Fertl. Steril. 1991; 56: 827-830.
4. Dubuisson, J.B., Chavet, X., Chapron, C., et al. Uterine rupture during pregnancy after laparoscopic myomectomy. Hum. Reprod. 1995; 10: 1475-1477.

5. Friedman, W., Maier, R.F., Luttkus, A., et al. Uterine rupture after laparoscopic myomectomy. Acta. Obstet. Scand. 1996; 75: 683-684.

6. Vercellini, P., Naddalena, S., et al. Determinants of reproductive outcome after abdominal myomectomy for infertility Fertil. Steril. 1999; 72: 109-114.

7. Dubuisson, J.B., Chapron, C., Chavet C. and Gregoralkis, S.S. Fertility after laparoscopic myomectomy of large intramural myomas: Preliminary results. Hum. Reprod. 1996; 11: 518-522.

8. Li T.C., Mortimer, R. and Cooke, I.D. Myomectomy: A retrospective study to examine the reproductive performance before and after surgery. Hum. Reprod. 1999; 14: 1735-1740.

9. Pelerme, G.R. and Friedman, E.A. Rupture of the gravid uterus in the third trimester. Amer. Obstet. Gynecol. 1996; 94: 571-576.

10. Pelosi, M.A.,III. and Pelosi, M.A. Spontaneous uterine rupture at thirty three weeks subsequent to previous superficial laparoscopic myomectomy. Amer. Obstet. Gynecol. 1997; 177: 1547-1549.

11. Hockstein, S. Spontaneous uterine rupture in the early third trimester after laparoscopically assisted myomectomy. J. Repro. Med. 2000; 45: 139-141.

12. Marret, H., Muriel, C. and Bruno, G. A retrospective multicentre study comparing myomectomy by laparoscopy and laparotomy in current surgical practice. What are the best patient selection criteria? Eur. J. Obstet. Gyn. Reprod. Biol. 2004; 117: 82- 86.

13. Seracchioli, R., Colombo, F.M., Bagnoli, A., et al. Laparoscopic myomectomy for fibroids penetrating the uterine cavity: is it a safe procedure? Int. J. Obstet. Gyn. 2003; 110: 236-240. 